\title{
Musculoskeletal Tuberculosis: A Multifaceted Foe
}

\author{
Uma Kumar $^{1}$ Sauvik Dasgupta ${ }^{1}$ \\ ${ }^{1}$ Department of Rheumatology, All India Institute of Medical \\ Sciences, Ansari Nagar, New Delhi, India
}

Ann Natl Acad Med Sci (India) 2019;55:3-5

\begin{abstract}
Address for correspondence Uma Kumar, MD, Department of Rheumatology, All India Institute of Medical Sciences, Room No. 4076 (A\&B), Fourth Floor, Teaching Block, Ansari Nagar, New Delhi 110029, India
\end{abstract}

\begin{abstract}
Keywords

- musculoskeletal tuberculosis

- sacroiliitis

- enthesitis

Tuberculosis (TB) still remains a global epidemic, and India accounts for one-fourth of the world's TB burden. The incidence of extrapulmonary TB has relatively remained constant, but with the introduction of antitumor necrosis factor, there has been a surge in pulmonary and extrapulmonary TB cases presenting to rheumatologists. Musculoskeletal TB accounts for 10 to $30 \%$ of all cases of extrapulmonary TB, with spondylitis (Pott's spine) being the most common manifestation. Manifestations mimicking autoimmune spondylitis are seen in $10 \%$ of cases. Tubercular arthritis most commonly presents with large joint monoarthritis, but oligo- or polyarticular involvement is also seen. Poncet's disease is a form of reactive arthritis occurring in patients with pulmonary or extrapulmonary TB, which is rarely seen with good response to antitubercular treatment. Quite often, there is delay in the diagnosis of musculoskeletal TB due to absence of constitutional symptoms. Treatment of musculoskeletal TB involves prolonged course of antitubercular treatment, and surgical interventions are limited to special cases.
\end{abstract}

\section{Introduction}

Tuberculosis (TB) still remains a global epidemic, killing 1.4 million people annually worldwide, with more than a third of the deaths occurring in India. ${ }^{1}$ India accounts for one-fourth of the world's ТВ burden, with an annual incidence of 28 lakhs and mortality of 4.8 lakhs for the year 2015. ${ }^{1}$ The incidence of extrapulmonary TB (EPTB) has relatively remained constant at 17 to $20 \%$ in the past decade in India. ${ }^{1}$ With the introduction of tumor necrosis factor inhibitors (TNFi) in 1999, there was a surge in both pulmonary TB (PTB) and EPTB, and an unexpectedly high number of TB cases started presenting to rheumatologists. This is particularly applicable to EPTB and disseminated TB, which showed a disproportionate increase in incidence on treatment with TNFi and other biologics. The percentage of EPTB among all TB cases in TNFi treated patients in various case series is as high as 57 to $62 \%{ }^{2,3}$

Musculoskeletal TB (MSKTB) comprises around 10 to 30\% of all EPTB cases. ${ }^{4,5}$ Diagnosis of MSKTB often becomes challenging due to its rarity at presentation and its often indolent presentation without typical features of fever and other constitutional symptoms characteristic of TB or other infections. Furthermore, it is complicated by the fact that tissue sample may not be always be accessible in case of skeletal TB, and less than $50 \%$ cases of MSKTB have features of present or past PTB. MSKTB is acquired hematogenously, or less commonly, by direct inoculation through trauma or local spread from infected surrounding structures such as lymph nodes. Clinically, MSKTB most commonly presents as tubercular spondylitis or Pott's spine (50-60\%). Other forms of presentation are osteomyelitis, septic arthritis, soft tissue abscesses, and rarely, reactive arthritis (Poncet's disease).

Spinal TB most commonly involves the lower dorsal or lumbar spine and most patients present with long-standing back pain with or without associated constitutional symptoms. This can often be confused with spondyloarthritis, especially considering the fact that sacroiliitis is seen in around $10 \%$ cases of spinal TB. Infections such as TB and brucellosis should always be kept in the diagnostic consideration when dealing with a case of unilateral sacroiliitis. Tubercular spondylitis can have extra-articular extension into surrounding structures causing soft tissue cold abscesses such as psoas abscess or retropharyngeal abscess. Tubercular spondylitis 
most often starts in the anterior vertebral body and may later spread to involve posterior spinal elements and intervertebral discs. This may lead to anterior wedge vertebral collapse and gibbus formation. The more dreaded complication of neurologic involvement in the form of myelopathy, radiculopathy, or cauda equina syndrome is seen in around 30\% of the cases and may need urgent intervention with stabilization of the spine. The American Spinal Injury Association (ASIA) Impairment Scale can be used to document the degree of neurodeficit in Pott's spine, with increasing severity of neurologic compromise from ASIA (E) to ASIA (A).

Apart from the vertebral body, tubercular osteomyelitis can involve other bones in the body too, especially the long bones such as femur and tibia. Tubercular osteomyelitis generally starts in the metaphysis and may spread contagiously to involve the adjacent joint such as hip or knee. Less commonly, involvement of ribs, pubic symphysis, phalanges, and skull can also be seen. Patients typically present with bone pain. Other associated symptoms may be localized swelling, abscess, or sinus formation. Although TB osteomyelitis is mostly unifocal, rarely it can present as multifocal osteoarticular TB with destructive lesions of multiple bones and joints.

Tubercular arthritis most commonly presents as large joint monoarthritis involving the hip or knee, although practically any joint of the body including sacroiliac joint, costotransverse joint, ankle, shoulder, elbow, and small joints of hand and feet can be involved. Tubercular oligo- or polyarthritis is less common and may be seen in around 10\% of the cases. Tubercular arthritis can be destructive in nature if antitubercular therapy (ATT) is not instituted early in the course of disease. Prosthetic joint TB infections have rarely been reported.

Poncet's disease is a rare form of acute-onset symmetrical inflammatory oligo- or polyarthritis involving commonly the knees, ankles, elbows and wrists. It is associated with active PTB or EPTB. Poncet's disease does not involve direct TB invasion of joints and is a form of reactive arthritis, and, therefore, TB bacilli cannot be demonstrated in the involved joints. It is quite rare, and to date, around 200 cases of Poncet's disease have been reported, 70 of which are from India. ${ }^{6}$ Unlike tubercular arthritis, Poncet's disease is a form of nondestructive arthritis and has a very rapid response to initiation of ATT. A similar kind of presentation has also been reported in patients treated with intravesical Bacillus Calmette-Guerin for bladder malignancy. ${ }^{7}$

Other forms of MSKTB include mostly abscesses in the form of epidural abscess or soft tissue abscess such as psoas abscess or retropharyngeal abscess. Soft tissue and osteoarticular involvement of hand may rarely give rise to dactylitis in TB.

Imaging may guide us in suggesting a diagnosis of $\mathrm{TB}$ and also help in delineating the extent of disease, although there are no pathognomonic imaging findings in MSKTB. Conventional radiography may show skeletal and soft tissue changes in cases of well-established disease. In Pott's disease, radiological abnormalities start in the anterior vertebral body and progress later to involve the posterior segments of the vertebra and intervertebral discs. Changes commonly seen on X-rays are loss of definition of bony margin, osteolysis, gibbus deformity, disc space narrowing and obliteration, and paraspinal abscess and calcification. Most cases involve lower dorsal or lumbar vertebra, with involvement of contiguous segments. Unilateral sacroiliitis can be seen in around 8 to $10 \%$ of cases. TB osteomyelitis lesions mostly appear as cystic or lytic lesions in metaphyses of long or flat bones. Tubercular bony lesions can be destructive, and the presence of lytic lesions may often lead the physician into suspicion of a malignancy. ${ }^{8}$ The radiographic findings in tubercular arthritis demonstrate periarticular osteopenia, peripheral osseous erosions, and joint space narrowing (Phemister's triad). A chest X-ray must always be performed in suspected cases of MSKTB to demonstrate the presence of typical lesions of PTB, if present, although less than $50 \%$ of cases show involvement of the lung. Compared with conventional radiography, magnetic resonance imaging (MRI) is much more sensitive to detect bony and soft tissue lesions. MRI helps in the early detection of TB changes in bone, with better visualization of the extent of disease and soft tissue spread. MRI has become an indispensable tool in cases of Pott's spine for the detection of spinal cord compression and neurologic involvement. Other imaging modalities such as CT scan, myelography, and scintigraphy can be used too for the detection of TB. CT scan is often used to obtain imaging-guided biopsy from the bone, synovium, or soft tissue.

The tuberculin skin test or Mantoux test is one of the oldest tests for $\mathrm{TB}$ and is still used as one of the screening tests for both PTB and EPTB. In rheumatological practice, it is also used as a screening test for detecting latent TB infection before the initiation of TNFi or other biologics. It is neither sensitive nor specific for the diagnosis of TB and only has an ancillary role in establishing a diagnosis of TB.

Staining of synovial fluid and other tissues for acid-fast bacilli by Ziehl-Neelsen staining has very poor sensitivity in cases of MSKTB, with being positive in only around 15 to $20 \%$ cases. Histopathology and culture are most often used for diagnosis. Synovial tissue culture demonstrates a higher sensitivity than synovial fluid culture and must be obtained in cases of suspected tubercular arthritis if synovial fluid assay is noncontributory. Histopathology of the synovium or bone may demonstrate caseating granuloma with or without acidfast bacilli. Cultures can also be obtained from infected fluids such as synovial fluid, cold abscess, or draining sinuses. Due to the slow-growing nature of Mycobacterium tuberculosis, culture results may take up to 42 days to yield results.

Newer methods to help in the diagnosis of TB include interferon- $\gamma$ release assays (IGRAs) and nucleic acid amplification techniques (NAATs). IGRA involves various methods that measure mononuclear cell interferon- $\gamma$ production on stimulation with tubercular antigens. In India, the method used is QuantiFERON-TB Gold (Qiagen). It is also commonly used for latent TB screening and can be repeated annually in patients on biologic therapy for checking IGRA conversion. NAATs provide a rapid, sensitive, and specific method of TB diagnosis, with a sensitivity of more than $85 \%$ and a specificity of $100 \%$ in cases of MSKTB. Nested or multiplex polymerase chain reaction (PCR) methods have now mostly been replaced 
in most places by Gene Xpert MTB/RIF, which, in addition to $\mathrm{PCR}$, also gives information on rifampicin resistance in diagnosed cases. In spite of added advantages, availability and cost limit its use in resource-constraint countries like India.

Treatment of MSKTB involves prolonged courses of ATT, with surgical intervention reserved for special cases. The Revised National Tuberculosis Control Program (RNTCP) recommends a 2-month intensive phase with isoniazid $(H)$, rifampicin (R), pyrazinamide, and ethambutol (E) followed by 4 months of continuation phase with HRE for newly diagnosed cases just like PTB. However, in cases of MSKTB, the continuation phase might be extended by 12 to 24 weeks depending on the clinical decision of the treating physician based on response. The RNTCP is currently moving from its thrice weekly ATT regimen to daily regimen. It has already been implemented for pediatric TB and TB with HIV. For adult $\mathrm{TB}$, it has been started initially in 104 districts. ${ }^{9}$ Most cases of MSKTB need treatment for 9 to 15 months for complete cure of the disease. The appearance of multidrug-resistant TB (MDR-TB) and extensively drug-resistant TB has posed new challenges in their treatment and requires prolonged courses of multiple second-line agents, sometimes for 30 to 36 months. $^{1}$

Surgical intervention is reserved for patients with Pott's spine with new or progressive neurodeficit or severe spinal deformity and in cases of large or nonresponding cold abscesses. Surgery can also be performed in undiagnosed cases for large tissue samples to aid in diagnosis. A wide variety of options such as decompression, radical focal debridement, and instrumental spine stabilization exist, and it is important to individualize the procedure on a case-to-case basis. Surgical debridement may also be helpful in MDR-TB cases. In cases of peripheral arthritis, total knee or hip arthroplasty might be undertaken only after successful completion of ATT. It is advisable to perform surgery after a prolonged gap, preferably more than a year after completion of therapy. In cases, where adequate gap cannot be given, ATT is continued for 3 months after surgery. Tissue should be obtained during surgery for TB culture, and prosthetic joint infection is uncommon if the intra-operative tissue is culture negative.

Quite often, there is delay in the diagnosis of MSKTB due to absent constitutional symptoms and its oligo- or polyarticular presentation. Awareness and high index of suspicion can avert this delay in a vast majority of patients.

\section{Conflict of Interest}

None declared.

\section{References}

1 Central Tuberculosis Division, Government of India. Available at: http://tbcindia.gov.in/WriteReadData/tbIndia2017.pdf. Accessed July 10, 2019

2 Keane J, Gershon S, Wise RP, et al. Tuberculosis associated with infliximab, a tumor necrosis factor alpha-neutralizing agent. N Engl J Med 2001;345(15):1098-1104

3 Dixon WG, Hyrich KL, Watson KD, et al; B S R B R Control Centre Consortium; BSR Biologics Register. Drug-specific risk of tuberculosis in patients with rheumatoid arthritis treated with anti-TNF therapy: results from the British Society for Rheumatology Biologics Register (BSRBR) Ann Rheum Dis 2010;69(3):522-528

4 Sharma SK, Mohan A. Extrapulmonary tuberculosis. Indian J Med Res 2004;120(4):316-353

5 Peto HM, Pratt RH, Harrington TA, LoBue PA, Armstrong LR. Epidemiology of extrapulmonary tuberculosis in the United States, 1993-2006. Clin Infect Dis 2009;49(9):1350-1357

6 Rueda JC, Crepy MF, Mantilla RD. Clinical features of Poncet's disease. From the description of 198 cases found in the literature. Clin Rheumatol 2013;32(7):929-935

7 Macía Villa C, Sifuentes Giraldo W, Boteanu A, González Lanza $\mathrm{M}$, Bachiller Corral J. Reactive arthritis after the intravesical instillation of BCG. Reumatol Clin 2012;8(5):284-286

8 Tsay MH, Chen MC, Jaung GY, Pang KK, Chen BF. Atypical skeletal tuberculosis mimicking tumor metastases: report of a case. J Formos Med Assoc 1995;94(7):428-431 